\title{
Biomimetic Fabrication of 3D Structures by Spontaneous Folding of Tapes
}

\author{
Derek A. Bruzewicz, Mila Boncheva, Adam Winkleman, \\ Jason M. St. Clair, Gregory Engel, and George M. Whitesides* \\ Department of Chemistry and Chemical Biology, \\ Harvard University, Cambridge, Massachusetts 02138 \\ *E-mail: gwhitesides@gmwgroup.harvard.edu
}

Supporting Information

Fabrication. We fabricated the flat tapes by using Mylar sheets (polyester film type C, gauge 10, a gift from DuPont Teijin Films) that were $2.5 \mu \mathrm{m}$ thick. Thicker Mylar sheets (polyester film type C, gauge 48, $12 \mu \mathrm{m}$ thick) were used to make the crimped tapes, since these sheets stayed creased and provided structural stability when crimped. After cleaning the sheets with water, detergent, and ethanol, we attached them to rigid supports (microscope glass slides or silicon wafers) using carbon tape and deposited successively $10 \mathrm{~nm}$ of chromium or nickel and $100 \mathrm{~nm}$ of copper using thermal evaporation. We patterned the metal layers in features that were intended to support solder using a combination of photolithography and wet etching as previously described(Boncheva, Ferrigno 
et al. 2003). We etched the copper and, where applicable, nickel layers (using saturated aqueous $\mathrm{FeCl}_{3}$ ); chromium gave a light grey color to the transparent tapes and allowed more contrast in optical imaging. After cutting the sheets with a razor blade to form patterned tapes, we crimped the tapes between two aluminum presses (combs) with complementary 3D shapes. We used water or ethanol to wet the comb that bore recessed features, and then we attached on end of the polymer tape to this comb with an adhesive tape. We crimped the polymer tape by applying slight pressure with the second, dry comb. Capillary interactions that involved the thin layer of water between the metal surface and the polymer tape held the crimped tape to the first, recessed comb after removal of the second one. While the tape remained in the recessed comb, we used a Qtip to apply a thin layer of glue (a gift from Exxon Chemical Corporation, containing a 6:4 mixture of styrenic block copolymers Vector@ and tackifying resin Escorez@ in toluene) onto the flat regions of the crimped tape. We then glued the flat tape to the crimped one. After allowing the glue to dry overnight, we removed the structure from the comb and covered the copper features on the crimped tape with solder (LMA-117 from Small Parts, Inc., melting temperature $47^{\circ} \mathrm{C}$ ) by dip-coating, as previously described(Gracias, Tien et al. 2000).

Fabrication of the tapes for the functional device was identical, except for the following changes. We patterned the thin, uncrimped tape with copper wires (100-200 nm thick, with $10 \mathrm{~nm}$ nickel adhesion layer underneath) by photolithography. We dipped IR-sensitive photodiodes (PDB-C171SM, Photonic Detectors Inc., Simi Valley, CA) in low-melting temperature solder, and placed 
these photodiodes roughly on the copper wires flat tape. After gently heating the tape on a hotplate $\left(\sim 50^{\circ} \mathrm{C}\right)$, the solder melted and fused the photodiodes in place. We applied a thin layer of the glue along the the top and bottom edges (the edges that did not form connections via solder) of each diode on the tape to prevent detachment during self-assembly. We then glued the two tapes together and allowed them to dry.

Self-assembly. We suspended the precursors in distilled water in a $20-\mathrm{mL}$ cylindrical, polystyrene container. We adjusted the $\mathrm{pH}$ of the water to 3 with $1 \mathrm{~N}$ $\mathrm{HCl}$ to dissolve any oxides formed on the surface of the solder; the water also contained a drop of detergent (Triton-X) to prevent formation of air bubbles on the walls of the container and on the tapes. We heated the water to approximately $60^{\circ} \mathrm{C}$ on a hot plate, and gently agitated the system by pipetting hot water at the unfolded precursor structures. Collisions between neighboring features carrying droplets of molten solder led to fusion of the droplets due to capillary interactions, and the tapes folded into the target structures. Cooling the suspension to room temperature solidified the solder and gave mechanical stability to the structures. We performed each self-assembly experiment at least 4 times. All precursors correctly folded into their target shapes. In all cases, folding completed within 2-5 minutes.

Testing the light-detector. After assembly, we dipped copper wire in lowmelting temperature solder and contacted the solder, while molten, with the solder-bearing edges of two light-detecting diodes on the assembled device. These copper leads, a red light-emitting diode and a p-junction JFET 
(MMBF5103, Fairchild Semiconductor, South Portland, ME) were mounted on an electrical breadboard as shown in Supplemental Figure 3. When illuminated with a hand-held laser, current flowed through the light-detecting diode. This current released the gate voltage that blocked current from passing through the lightemitting diode, and red light was emitted. 


\section{Supplemental Figure 1}

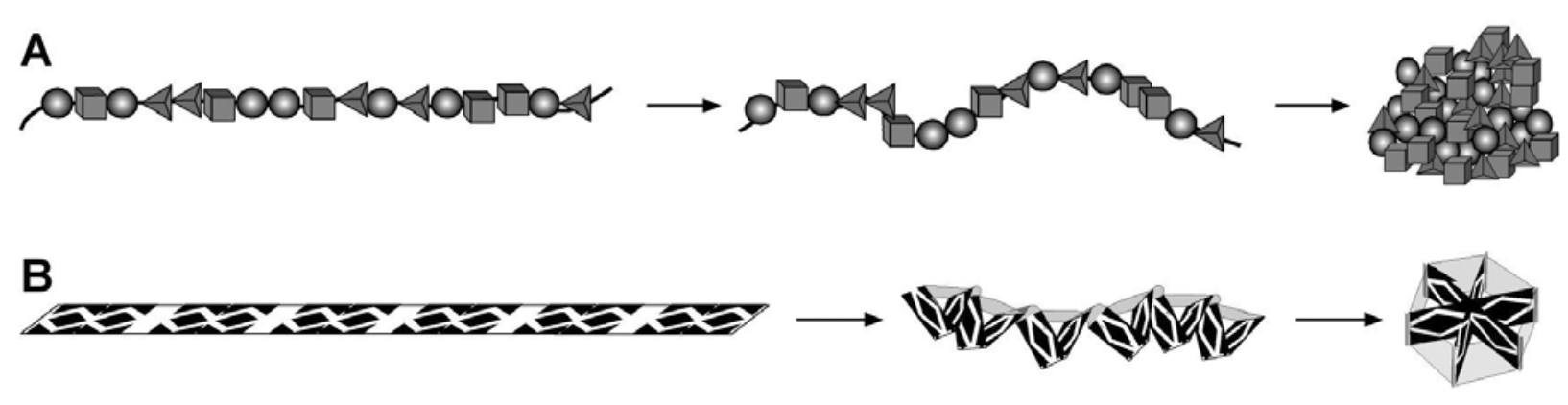

Supplemental Figure 1. A scheme illustrating the "beads-on-a-string" concept in folding of biological macromolecules (A) and flexible tapes (B). In both examples, unfolded precursors containing linear sequences of monomers (e.g., amino acid residues or 3D polyhedra) fold into 3D structures; the type of structures that form depends on the initial sequence of the monomers and on the interactions between them. 


\section{Supplemental Figure 2}

Supplemental Figure 2. Fabrication of the light-detector. 

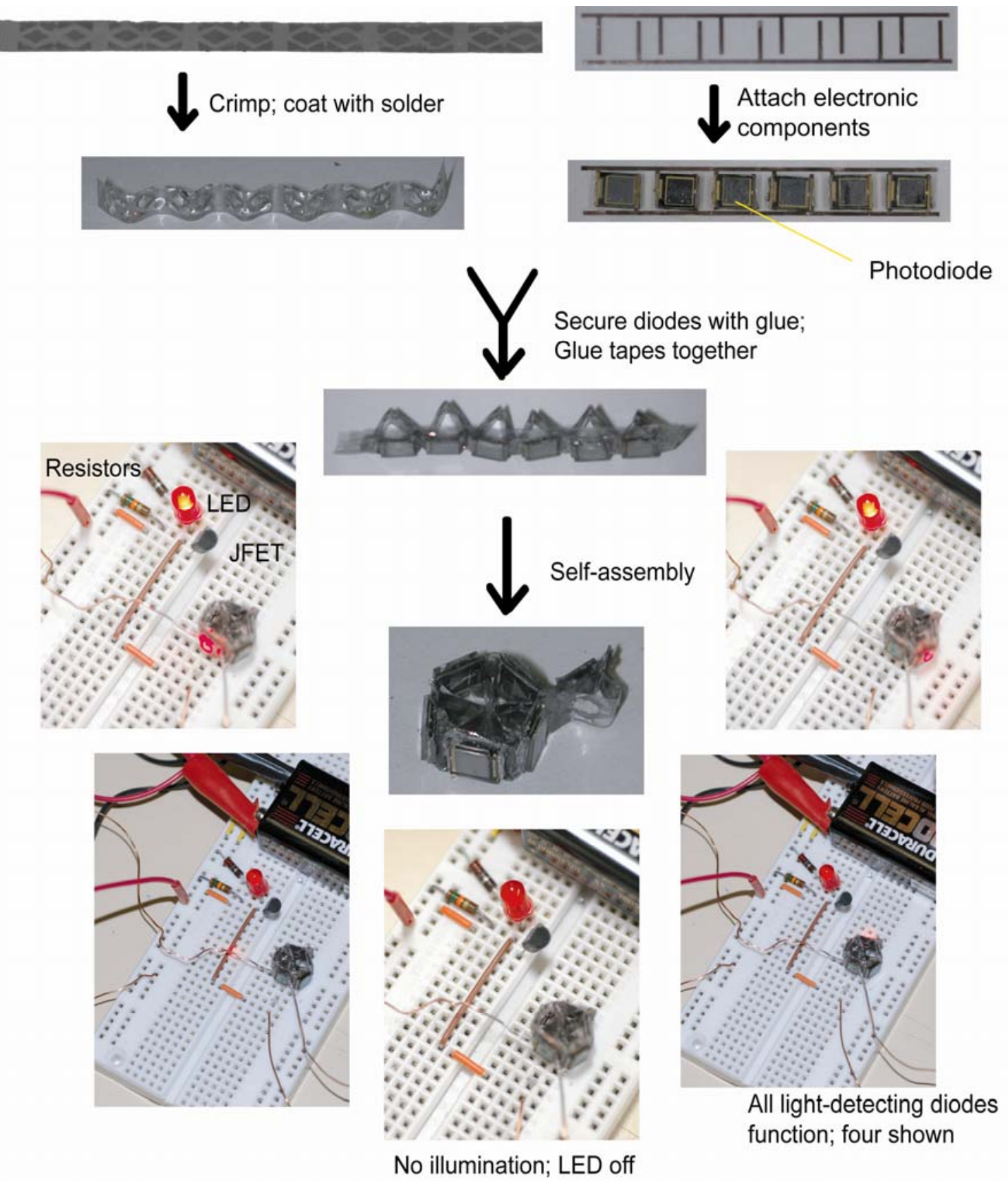
Figure 3

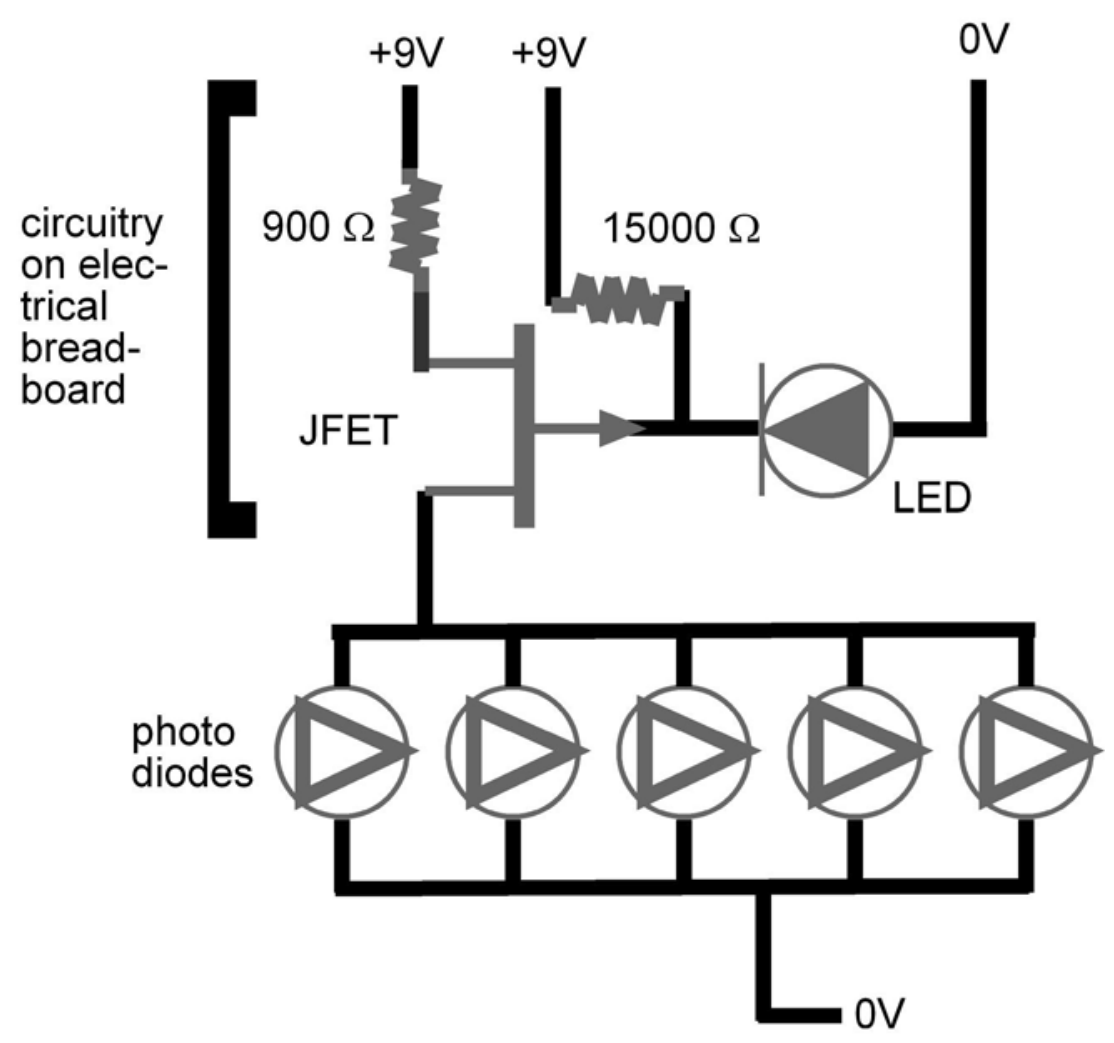

Supplemental Figure 3. A schematic diagram of the design of the lightdetector. Illumination of the photodiode with 650-nm light from a diode laser allows current to flow through it to ground, and simultaneously removes the positive gate voltage from the $p$-channel JFET; removal of the gate voltage allows current to flow through the LED, thereby illuminating it. 


\section{References}

Boncheva, M., R. Ferrigno, et al. (2003). "Plasticity in Self-Assembly: Templating

Generates Functionally Different Circuits from a Single Precursor." Angew. Chem. Int.

Ed. 42(23): 2644-2647.

Gracias, D. H., J. Tien, et al. (2000). "Forming Electrical Networks in Three Dimensions by Self-Assembly." Science 289: 1170-1172. 